\title{
Distribution characteristics and health risk assessment of PAEs in urban soils of Changji City, Xinjiang, China
}

\author{
Liu Y. ${ }^{*}$, Ji C. ${ }^{1}$, Yu Y. ${ }^{2}$, Liu H. ${ }^{3}$ and Shen Y. ${ }^{2}$ \\ ${ }^{1}$ School of Geography and Environmental Science, Hainan Normal University, Haikou, Hainan 571158, P. R. China \\ ${ }^{2}$ Department of Chemistry and Applied Chemistry, Changji University, Changji 831100, P. R. China \\ ${ }^{3}$ Changji Environmental Monitoring Station, Changji 831100, P.R. China \\ Received: 10/10/2019, Accepted: 16/12/2019, Available online: 16/01/2020 \\ *to whom all correspondence should be addressed: e-mail: cjenvironment@126.com \\ https://doi.org/10.30955/gnj.003230
}

\begin{abstract}
Recent studies have indicated that PAEs (phthalic acid esters) are significant environmental pollutants with carcinogenic, teratogenic, and mutagenic effects. Urban soils can act as both a source and sink for city contaminants. This paper takes Changji, Xinjiang in Northwest China as the study area and explores the pollution characteristics and health risks of PAEs in the urban soils of arid areas. The analysis results indicate average concentration occurrences of $13.77 \mathrm{mg} / \mathrm{kg}$ and ranges from 0.29 to $119.72 \mathrm{mg} / \mathrm{kg}$ in the study area. Dibutyl phthalate (DBP) and di-(2-ethylhexyl) phthalate (DEHP) were the predominant PAEs in the urban soil of Changji. The highest average PAEs concentration appeared in winter, followed by autumn and spring and the lowest in summer; the seasonal variation was significant. The climate characteristics of arid regions and seasonal variations of source emissions were the primary influencing factors. The theoretical values of the non-carcinogenic and carcinogenic risk of PAEs were relatively small, all within the acceptable range. Values of non-carcinogenic risk and carcinogenic risk to children were much higher than the risks to adults. Children were the vulnerable groups most at risk for harmful pollutants, and the safety of their living environment should be the focus of more attention.
\end{abstract}

Keywords: Urban soil, PAEs, pollution characteristics, health risk.

\section{Introduction}

Phthalic acid esters (PAEs) are widely used in industrial production. PAEs are often used as plasticizers in the production of plastic to increase product plasticity and improve product strength. Additionally, PAEs compounds are used in the production of rubber, pesticide carriers, coatings, insect repellents, cosmetics, lubricants, defoamers, etc. PAEs and plastics bond via hydrogen bonding or van der Waals force, so the stability of the resulting product is relatively poor. In the production, use and final disposal of plastic products, PAEs can easily migrate from the plastic to the environment (Al-Natsheh et al., 2015; Kong et al., 2015). With the extensive application of plastic products, PAEs are a significant emission and widespread in the air, water, soil, sediment, biological and other environmental media (Kranich et al., 2013; Kong et al., 2015; Paluselli et al., 2018). The study also found that coal combustion can also release PAEs (Wang et al., 2015a). Recent studies have indicated that PAEs are significant environmental pollutants with carcinogenic, teratogenic, and mutagenic effects. PAEs are uniquely toxic to reproductive systems in humans and other animals. They are universally capable of interfering with normal endocrine activity, which leads to an abnormal reproductive system and behaviour. Six of the most widely used PAEs are classified as priority pollutants by the US Environmental Protection Agency (USEPA), and of these, three types have also been listed as environmental priority control pollutants in China (USEPA, 2013; Yang, 2013). The environmental behaviour of PAEs has become a hot topic in related fields.

PAEs are hydrophobic organic pollutants that are easily distributed to soil organic matter. PAEs continuously migrate in the environment through wet and dry deposition, sewage discharge, accumulation of waste plastic products, etc. and finally accumulate in the soil. It has been shown that PAEs are one of the most abundant in soil organic contaminants (Niu et al., 2014). Urban soils are a central part of the urban environment and can act as both a source and sink for urban environment pollutants. In the current accelerating process of urbanization, urban soil pollutants are not only a complex source of pollutants, but the pathways of human toxicity have become more complex. The primary routes for urban soil contaminants to enter the body include hand-mouth contact intake, respiratory inhalation and skin absorption ( $\mathrm{Li}$ and Liu, 2007). The potential ecological risks of PAEs pollution in urban soils should not be neglected, given their concealed and persistent risk in intensive urban human activities (Xia et al., 2011).

Human activities and natural conditions severely affect the fates of environmental pollutants. Arid regions in Xinjiang have a special natural environment, i.e., rare precipitation, significant evaporation, more wind and sand weather, large 
temperature differences between day and night. The urban soils in arid regions also have the characteristics of strong alkalinity and rich phosphorus content (Li and Liu, 2007). Most studies on soil PAEs focus on cropland systems, and less on urban soils (Zeng et al., 2009; Niu et al., 2014; Li et al., 2015; Sun et al., 2016; Wang et al., 2015b). At present, the environmental behavior of urban soil PAEs and its influencing mechanism are still unclear, and relevant research on arid areas have not been reported. Therefore, the characteristics of PAEs pollution and its health risk assessment in urban soils in Xinjiang arid region have certain theoretical value and regional characteristics.

\section{Materials and methods}

\subsection{Overview of research area}

Changji $\left(86^{\circ} 24^{\prime}-87^{\circ} 37.0^{\prime} \mathrm{E}, 43^{\circ} 06^{\prime}-45^{\circ} 20^{\prime} \mathrm{N}\right)$ is in the northwestern part of China, in the centre of the Eurasian continent and is a relatively new city. As the satellite city of Urumqi (capital of Xin jiang, China), it has a typical continental arid climate. Recently, the Chinese government's western development policy has promoted the rapid urbanization. In this paper, Changji City was taken as the study area. Dimethyl phthalate (DMP), diethyl phthalate (DEP), di-n-butyl phthalate (DBP), di(2-ethylhexyl) phthalate (DEHP) were selected as the target pollutants, which were listed as priority pollutants by the USEPA and have relatively high detectable contents in environmental medium. This study aimed to investigate the PAEs pollution characteristics and health risks in urban soils.

\subsection{Sample collection}

Sampling was based on urban functional areas and the microenvironment in Changji City. According to land uses, the city was divided into heavy traffic, industrial and residential/educational areas. Five sampling points were selected and arranged in each of the above three functional areas, and a total of 15 sampling points were set. The $0-2$ $\mathrm{cm}$ part of the surface layer of the soil was collected. Each point adopted the multi-point mixing method. The specific collection situation of the multi-point mixing method was as follows: Set a center point for each sampling point, and 6-7 point samples were collected around the center point, and then equal and uniformly mix to represent a sample. The soil samples were collected month by month and 15 samples per month were collected. There are 180 samples which were collected for this research. The sampling period was from March 2016 to February 2017. In the sampling process, sampling was conducted to avoid any plastic products and simultaneous recordied meteorological conditions, traffic, flow and other sampling microenvironment.

\subsection{Sample analysis}

\subsubsection{Main instruments and reagents}

The main instruments were as follows: a SHIMADZU GC2010 gas chromatograph (GC), a RE-52AA rotary evaporator and HY-CXJ-type high-power ultrasonic cleaner. Four pure standard samples of DMP, DEP, DBP, DEHP and surrogate standards, consisting of $500 \mathrm{~g} \cdot \mathrm{ml}^{-1}$ each of DBP and DEHP (Aldrich Chemicals, Gillingham, UK) were used. Benzyl benzoate, which was used as the internal standard, was acquired as a solid of $99 \%$ purity (Aldrich Chemicals, Gillingham, UK). Anhydrous sodium sulfate was baked at $420{ }^{\circ} \mathrm{C}$ for $12 \mathrm{~h}$ and stored in sealed glass jars. The filter paper was extracted with methanol and $n$-hexane with the Soxhlet extractor for $72 \mathrm{~h}$ before use. Water was filtered by Milli-Q and double distilled. All organic solvents used were of analytical grade and redistilled using a glass system. Laboratory glassware was soaked overnight in $\mathrm{K}_{2} \mathrm{CrO}_{7} / \mathrm{H}_{2} \mathrm{SO}_{4}$ solution, washed with tap water and redistilled water, baked at $300{ }^{\circ} \mathrm{C}$ for $12 \mathrm{~h}$, and then rinsed with acetone and $n$-hexane.

\subsubsection{Experimental methods}

The samples were dried in a dry, ventilated place and passed through a 40-mesh screen to remove impurities and large particles and then passed through a 200-mesh sieve after drying to be extracted. Each soil sample was weighed to $2 \mathrm{~g}$ using an analytical balance. The samples were wrapped in qualitative filter paper and placed in a Soxhlet extractor. The samples were extracted with hexane and acetone (1:1) for $8 \mathrm{~h}$ with the temperature controlled at $74.5{ }^{\circ} \mathrm{C}$. The resulting solution was extracted with Floris cartridges to remove macromolecular organics and impurities. Moreover, the filtered solution was dried with nitrogen. Normal hexane was added to give a $1 \mathrm{~mL}$ test solution.

The PAEs were determined using Hydrogen Flame Gas Chromatography. The working conditions of the GC were as follows: the temperature of the vaporization chamber was $260^{\circ} \mathrm{C}$, the detector was a hydrogen flame ionization detector (FID) at a temperature of $260{ }^{\circ} \mathrm{C}$. The nitrogen carrier gas, hydrogen gas, and air (all $\geq 99.999 \%$ ) were kept at constant speeds of $30 \mathrm{~mL} / \mathrm{min}, 40 \mathrm{~mL} / \mathrm{min}$, and 400 $\mathrm{mL} / \mathrm{min}$, respectively. The extracted samples were injected into the GC in split mode with a split ratio of 9:1. The injection volume was $1.0 \mu \mathrm{L}$, and the temperature was increased from $120^{\circ} \mathrm{C}$ to $260^{\circ} \mathrm{C}$.

\subsubsection{Quality assurance and quality control}

The results showed a good linear relationship, and the correlation coefficients of the four PAEs were all greater than 0.998. The detection limits of the DMP, DEP, DBP and DEHP standards were between 0.008 and $0.025 \mathrm{mg} / \mathrm{L}$. To test the method precision, the determination of the 50 $\mu \mathrm{g} / \mathrm{mL}$ mixed standard solution concentration was repeated five times. Among them, the relative deviations of DMP, DEP, DBP and DEHP were $2.0 \%, 1.6 \%, 2.2 \%$ and $4.2 \%$ respectively, both less than $5 \%$. To ensure the reliability of the experimental data, the same sample was used for parallel determination, with a deviation threshold not to exceed $5 \%$.

\section{Results and discussion}

\subsection{Concentration of PAEs and its influencing factors}

When only these four PAEs were monitored, the average PAE concentration in the Changji City soil was $13.77(0.29-119.72) \mathrm{mg} / \mathrm{kg}$. Among them, average concentrations of DMP, DEP, DBP and DEHP in the soil were 
0.87(ND -5.75) mg/kg, 1.53 (ND -11.18) mg/kg, $9.72(0.15-$ $102.36) \mathrm{mg} / \mathrm{kg}, \quad 1.66$ (ND-15.12) $\mathrm{mg} / \mathrm{kg}$. Respectively (Figure 1). DBP detected the highest proportion of four kinds of PAEs, followed by DEHP, and DMP content is relatively minimum. Relative to other PAEs, The total amount of DBP and DEHP are the most commonly used plasticizers, accounting for $50-60 \%$ of all plasticizers (Xia et al., 2011; Adeniyi et al., 2011). Thus, the emissions of these two PAEs in the urban environment are relatively large. Compared with other PAEs, DBP and DEHP belong to middle and high molecular weight PAEs with longer molecular chain, larger octanol-water partition coefficient and weaker activity. These PAEs are not easily degraded by microorganisms in the soil and do not migrate as quickly via runoff and leaching and hence rapidly accumulate in the soil. DMP, DEP molecular weight is relatively small, short alkyl chain, the migration speed is faster and more volatile (Zeng et al., 2009; Niu et al., 2014; Wang et al., 2015b; Langer et al., 2010). These factors might be the reason why DBP and DEHP were the primary PAE pollutants in the urban soil.

Presently, there is no control standard for PAE soil pollution in China. Therefore, this study was based on the control criteria for PAEs in the United States soils (USEPA, 2013). In this study regions, DMP, DEP, DBP and DEHP in the urban soil samples exceeded this standard by varying degrees, DMP, DEP and DBP were exceeded the standard serious, particularly DBP which exceeded the standard by three orders of magnitude. Although the DMP, DEP and DEHP concentrations did not exceed the United States governance standards, DBP significantly exceeded the governance standards. PAEs pollution problems should arouse the concern of the relevant management (Table 1).

The PAEs content found in Changji was much higher than that in other cities in China, e.g., Beijing, Tianjin and Anshan (Table 1). The sampling time for Changji City was later than that in the other cities, so PAEs had accumulated in the soil for a longer time. Additionally, the Changji samples were collected during four seasons, so these PAE concentrations represent the annual average value. The samples from Beijing, Tianjin and Anshan were collected in summer, and these three cities are in temperate monsoon climate regions. In these regions, summer rainfall is plentiful and easily forms runoff. Runoff scouring exacerbates the migration of PAEs into the aquatic environment. Especially in summer, soil PAEs are more volatile, and these summer PAEs concentrations represented these cities' annual mean values, which might result in the artificially low PAE concentrations for these cities. The DEHP concentration in Guangzhou was about ten times that in Changji City. Guangzhou is an important industrial base in China and a comprehensive industrial manufacturing center in South China.

Light industry is more developed, cosmetics, plastics and rubber production and other industries scale were larger, This might be the main reason that led to the large emissions of overall PAEs.

It is found that in addition to DMP, the Changji urban soil PAEs content was much higher than that of domestic agricultural soils. This result is consistent with other studies (Xia et al., 2011; Zeng et al., 2009). Urban soils are characterized by a high organic matter content (Li and Liu, 2007). The soil organic matter content has a significant effect on the soil adsorption capacity of PAEs, which increases with the increase of organic matter content (Yang, 2013). Additionally, the PAEs in agricultural soil are primarily from sewage and agricultural plastic film, while the PAEs in an urban environment are more complex and their emissions larger. Wang et al. study also shows that the river bank deposits, PAEs content riverbank sediments in urban areas is much higher than suburban and rural (Sun et al., 2016; Wang et al., 2014). This discrepancy indicates that urbanization significantly increases PAE emissions in the local environments. This factor are also the primary reason that the PAE content in the urban soil was higher than that agricultural soil.

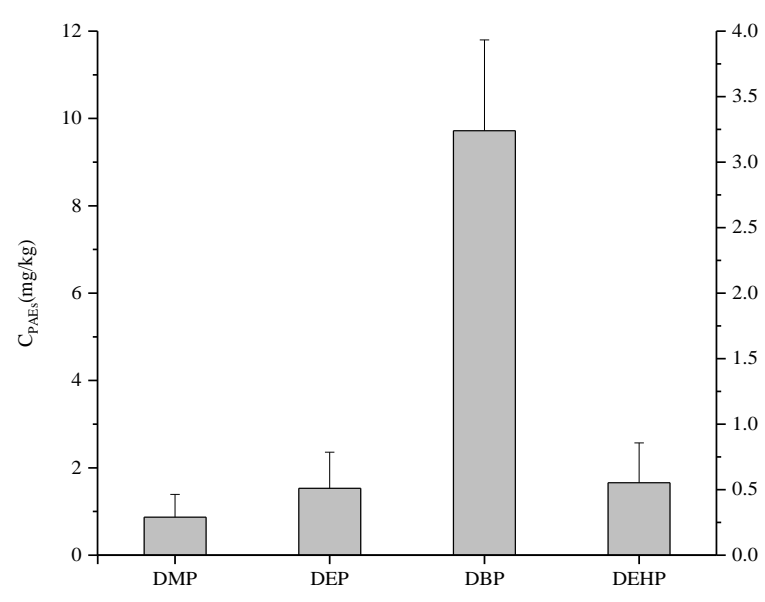

Figure 1. Concentration of PAES

\subsection{Seasonal variation of PAEs and its influencing factors}

The concentrations of $\Sigma_{4}$ PAEs in spring, summer, autumn and winter were $3.05 \mathrm{mg} / \mathrm{kg}, 2.19 \mathrm{mg} / \mathrm{kg}, 8.89 \mathrm{mg} / \mathrm{kg}$ and $46.76 \mathrm{mg} / \mathrm{kg}$, respectively. The concentration of $\Sigma_{4} \mathrm{PAEs}$ was highest in winter, followed by autumn and spring, and minimum content in summer (Figure 2). Four seasons change in Xinjiang are obvious, the winter was very cold, and there was a heating period during the autumn, winter and spring, starting from October of the year to the end of next year April, for up to six months or more. The primary heating method in Changji relies on coal combustion and the coal-burning process can release PAEs to the environment (Wang et al., 2015a). During the winter, the low temperatures were not conducive to PAEs volatilizing from the soil. Further, winter is the season with the least precipitation, and the precipitation was solid. Combined with the low temperatures, runoff erosion of soil PAEs and deep penetration to the soil does not easily occur during winter. These might be the reasons that the concentration of $\Sigma_{4}$ PAEs was highest in winter. 
Table 1. Distribution of PAEs in Chinese soils $(\mathrm{mg} / \mathrm{kg}$ )

\begin{tabular}{|c|c|c|c|c|c|c|c|}
\hline & Sampling Location & DMP & DEP & DBP & DEHP & Sample year & Data sources \\
\hline Control standards & & 0.02 & $7.10 \times 10^{-2}$ & $8.10 \times 10^{-2}$ & 4.35 & Soil types & (USEPA, 2013) \\
\hline Governance standards & & 2.0 & 7.1 & 8.1 & 50 & - & (USEPA, 2013) \\
\hline \multirow{5}{*}{ Urban soil } & Changji, Xinjiang & 0.87 & 1.53 & 9.72 & 1.66 & 2015 & This Research \\
\hline & Tianjin & $1.50 \times 10^{-3}$ & $6.67 \times 10^{-3}$ & 0.37 & 0.42 & 2008 & (Zhu et al., 2012) \\
\hline & Anshan, Liaoning & $6.67 \times 10^{-3}$ & $7.83 \times 10^{-3}$ & 0.59 & 0.43 & 2008 & (Zhu et al., 2012) \\
\hline & Beijing & $6.18 \times 10^{-3}$ & $1.27 \times 10^{-3}$ & 0.99 & 0.14 & 2008 & (Xia et al., 2011) \\
\hline & Guangzhou & - & - & 1.80 & 14.8 & 2005 & (Zeng et al., 2009) \\
\hline Agricultural soils & China & $1.48 \times 10^{-2}$ & $2.79 \times 10^{-3}$ & $6.58 \times 10^{-2}$ & $8.21 \times 10^{-1}$ & 2013 & (Niu et al., 2014) \\
\hline
\end{tabular}

Table 2. Health risk assessment parameters of PAEs

\begin{tabular}{|c|c|c|c|c|}
\hline Parameters & Physiological parameters & Unit & Value & Data sources \\
\hline $\mathrm{C}$ & Concentration of Soil PAEs & $\mathrm{mg} / \mathrm{kg}$ & Average value & This research \\
\hline IngR & Percentage of pathways through ingestion & $\mathrm{mg} / \mathrm{d}$ & 200 (child) 100 (adult) & \\
\hline $\operatorname{lnhR}$ & Inhalation frequency & $\mathrm{m}^{3} / \mathrm{d}$ & 7.63 (child) 20 (adult) & \\
\hline $\mathrm{EF}$ & Exposure frequency & $d / a$ & 180 & \\
\hline ED & Exposure time & $\mathrm{a}$ & 6 (child) 24 (adult) & \\
\hline SA & Exposure to skin surface area & $\mathrm{cm}^{2}$ & 2800 (child) 5700 (adult) & (USEPA, 2011) \\
\hline $\mathrm{SL}$ & Skin adhesion & $\mathrm{mg} / \mathrm{cm}^{2}$ & 0.2 (child) 0.07 (adult) & \\
\hline$A B S$ & Skin absorption factor & $1 / d$ & 0.1 & \\
\hline BW & Mean body weight & $\mathrm{kg}$ & 15 (child) 70 (adult) & \\
\hline AT & Mean exposure time & $d$ & ED $\times 365$ (non-carcinogenic), $70 \times 365$ (Carcinogenic) & \\
\hline PEF & Surface dust content factor & $\mathrm{m}^{3} / \mathrm{kg}$ & $1.3610^{9}$ & (Maxwell et al., 1978) \\
\hline $\mathrm{R}_{\mathrm{fd}}$ & Reference dose & $\mathrm{mg} / \mathrm{kg} \cdot \mathrm{d}$ & $8.00 \times 10^{-1}(\mathrm{DEP}), 1.0 \times 10^{-1}(\mathrm{DBP}), 2.00 \times 10^{-2}(\mathrm{DEHP})$ & (USEPA, 1996, 2002) \\
\hline SF & Carcinogenic slope & $(\mathrm{mg} / \mathrm{kg} \cdot \mathrm{d})^{-1}$ & $1.40 \times 10^{-2}(\mathrm{DEHP})$ & \\
\hline
\end{tabular}


During the spring and autumn, heating was just beginning or nearing completion, so less coal was burned, and PAE emissions were reduced. In addition, compared with winter, temperatures are relatively high in spring and autumn, and so did the volatilization of soil PAEs to the atmosphere. Compared with winter, there was an inevitable increase in precipitation in spring and autumn. Thus, the possibility of runoff formation also increases and causes runoff scouring and rainwater infiltration of PAEs into the soil. These factors raised the possibility of soil PAEs migrating to the water environment.

In summer, PAE emissions from coal-fired heating were missing. Concurrently, the highest temperatures occurred in Xinjiang, so the chance of PAEs volatilizing from the soil into the atmosphere are maximized. The precipitation also reached a maximum for the year, increasing runoff scouring and rainwater infiltration, which might be the reason for the occurrence of the lowest soil PAE concentrations in summer. The seasonal variation of DBP and DEHP was consistent with $\Sigma_{4}$ PAEs, but the seasonal variation of DMP and DEP was not. This discrepancy may be related to the characteristics and seasonal variations in relevant sources of DMP and DEP (Langer et al., 2010; Li et al., 2016). Hu et al. found that soil PAEs with lower and higher molecular weights occur in different states, with significant differences in migration, transformation and bioavailability (Hu et al., 2007).

Studies show that there are distinct seasonal variations of ¿PAEs in farmland (agricultural soil), but that concentrations are highest in summer, contrary to the seasonal variation of urban soil (Wang, 2015). There were a large number of plastic film left in the facility farmland. During summer, the temperature reaches the highest of the year, and the temperature of the plastic film in the soil. This factor leads to a substantial reduction of the bond strength between the plastic molecules and PAEs. The accelerated release of PAEs in the mulch film plays a major role in PAEs enrichment in the soil. The PAEs from differences sources was the reason why the variation of PAEs concentration seasonal variation in urban soil and farmland soil are primarily.

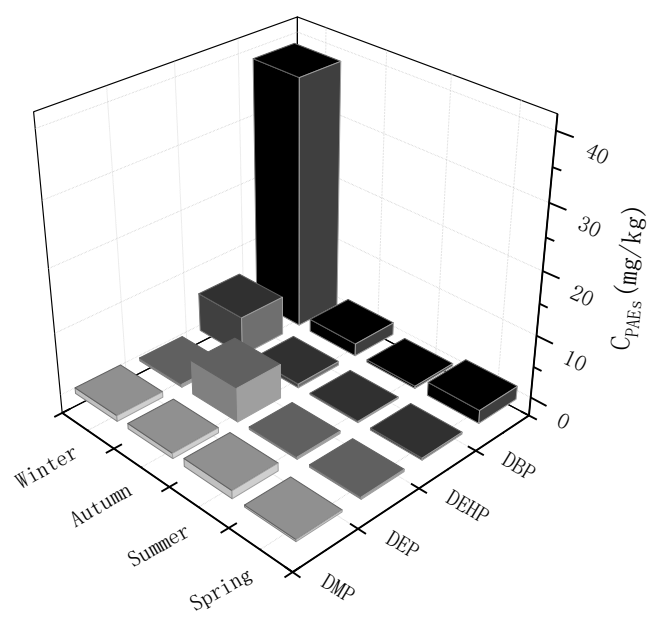

Figure 2. Seasonal variation of PAEs

\subsection{Health risk assessment}

To evaluate the toxicity of PAEs, scholars had evaluated the exposure and health risks of PAEs in the atmosphere, water and other environmental media (Niu et al., 2014; Wang et al., 2015b; Li et al., 2016; Bui et al., 2016). Among these evaluations, the risk assessment of soil is primarily for that in agricultural soil (Niu et al., 2014; Duan et al., 2010). There are few reports evaluating urban soils. Given the concealment and persistence of urban soil pollutants and the large urban population, it is urgent and necessary to conduct health risk assessments of PAEs in urban soils.

According to the migration cycles of urban soil surface pollutants in the environment, three exposure routes of urban soil PAEs to the human body were determined: hand-mouth contact intake, respiration inhalation, and direct skin absorption ( $\mathrm{Li}$ and Liu, 2007). The health risk assessment of urban soil in the study area was conducted using the US EPA human exposure risk assessment model (USEPA, 2004). Health risks include carcinogenic and non-carcinogenic risks. DEHP is one of the more toxic PAEs. The global harmonized system of classification and labelling of chemicals (GHS) defines two types of carcinogens: one class of substances includes known carcinogens, and the other class includes substances that are suspected carcinogens. DEHP has been classified in the Category known to be a carcinogenic substance (Chang, 2011). DEHP has been banned from use in child care supplies, toys, cosmetics and medical devices (Bui et al., 2016; Buzek and Ask, 2009). The high toxicity and low degradability of DEHP mean more focus should be on its health risk (Bui et al., 2016; Sampson and De Korte, 2011) The PAEs were divided into non-carcinogenic (DEP, DBP and DEHP) and carcinogenic (DEHP) categories; DEHP was classified as both a non-carcinogen and a carcinogen to evaluation it. There were no sound DMP evaluation parameters. Therefore, the evaluation in this study was only for DEP, DBP, and DEHP.

\subsubsection{Calculation of exposure dose}

The exposure dose was calculated according to the soil concentration and exposure route of PAEs as follows:

Hand-mouth contact intake:

$$
D_{\text {ing }}=C_{(\text {PAES })} \times \frac{\operatorname{lngR} \times E F \times E D}{B W \times A T} \times 10^{-6}
$$

Respiration inhalation:

$$
D_{\text {inh }}=C_{\text {(PAEs) }} \times \frac{\operatorname{lnhR} \times E F \times E D}{P E F \times B W \times A T}
$$

Direct skin absorption:

$$
D_{\text {der }}=C_{\text {(PAEs) }} \times \frac{S A \times S L \times A B S \times E F \times E D}{B W \times A T} \times 10^{-6}
$$

where $D$ is the exposure dose of each PAE and the other physiological parameters values are shown in Table 2 . 


\subsubsection{Non-carcinogenic risk assessment}

To obtain the noncarcinogenic contaminants Hazard Index, the non-carcinogenic contaminant exposure dose is divided by the reference dose:

$$
H I=\sum\left(\frac{D}{R_{f D}}\right)
$$

where $\mathrm{RfD}_{\mathrm{f}}$ is the reference dose (Table 2 ) and $\mathrm{HI}$ is the total non-carcinogenic hazard index, it meaning the integrated non-carcinogenic risks of the pollutants multiple exposure pathways. When the calculation result is less than 1 , the risk is considered low or negligible. If the result is greater than 1 , it believes there is a non-carcinogenic risk (USEPA, 2004).

Non-carcinogenic risk assessment results are shown in Table 3. For adults, the non-carcinogenic risk of PAEs in urban soils was DBP> DEHP> DEP, and children's risk was characterized as DEHP> DBP > DEP. Although the average non-carcinogenic risk values of the three PAEs were all less than 1, total non-carcinogenic hazard index of DEHP and DBP were relatively higher. However, the non-cancer risk from PAEs to children was greater than to adults by approximately an order of magnitude. The primary reasons for this difference are that children's physical characteristics and living habits make them more likely to be exposed to pollutants and they are more sensitive to pollutants. Although the theoretical non-carcinogenic risk values of PAEs are within acceptable range, they may still be harmful to the health of susceptible people, Especially the maximum risk of DBP and DEHP has exceeded $1 \times 10^{-3}$ for children. Children are even more Sensitive risk receptors, The safety of children's living environment should be the focus of attention.

\subsubsection{Carcinogenic risk assessment}

For carcinogenic substances, the exposure dose multiplied by the corresponding cancer slope factor (SF) results in the carcinogenic risk

$$
(\text { Risk })_{T}=\sum(D \times S F)
$$

where SF is the carcinogenic slope (Table 2) and (Risk) $\mathrm{T}$ is the total carcinogenic risk. This expression shows the variety of exposure pathways for pollutants in a comprehensive carcinogenic risk assessment and the probability of cancer in a population. (Risk) ${ }_{T}$ is not more than $10^{-6}$ for the upper limit of acceptable cancer risk (USEPA, 2004).

In the carcinogenic risk assessment shown in Table 3, the average carcinogenic risk for DEHP in adults and children was below $10^{-6}$, within the acceptable safety range. Meanwhile, the average carcinogenic risk for DEHP in children was much higher than that for adults. Specifically, the greatest cancer risk value was more than $10^{-7}$. Therefore, the DEHP may be a health hazard to children and other susceptible populations so the potential cancer risk should not be ignored.

\begin{tabular}{|c|c|c|c|c|}
\hline Child & & DEP & DBP & DEHP \\
\hline \multirow{2}{*}{ Non-carcinogenic risk } & Ave & $1.61 \times 10^{-5}$ & $\begin{array}{c}8.71 \times \\
10^{-4} \\
\end{array}$ & $\begin{array}{c}5.45 \times \\
10^{-4} \\
\end{array}$ \\
\hline & Max & $8.8 \times 10^{-4}$ & $\begin{array}{c}1.88 \times \\
10^{-3}\end{array}$ & $\begin{array}{c}1.39 \times \\
10^{-3}\end{array}$ \\
\hline \multirow{2}{*}{ Carcinogenic risk } & Ave & & & $\begin{array}{c}1.69 \times \\
10^{-8}\end{array}$ \\
\hline & Max & & & $\begin{array}{c}1.55 \times \\
10^{-7} \\
\end{array}$ \\
\hline Adult & DEP & DBP & DEHP & \\
\hline \multirow{2}{*}{ Non-carcinogenic risk } & Ave & $1.90 \times 10^{-6}$ & $\begin{array}{c}7.85 \times \\
10^{-5} \\
\end{array}$ & $\begin{array}{c}8.15 \times \\
10^{-5}\end{array}$ \\
\hline & Max & $1.78 \times 10^{-5}$ & $\begin{array}{c}1.01 \times \\
10^{-4} \\
\end{array}$ & $7.5 \times 10^{-4}$ \\
\hline \multirow{2}{*}{ Carcinogenic risk } & Ave & & & $\begin{array}{c}7.84 \times \\
10^{-9} \\
\end{array}$ \\
\hline & Max & & & $\begin{array}{c}7.00 \times \\
10^{-8} \\
\end{array}$ \\
\hline
\end{tabular}

Table 3. Health risk assessment of PAEs in urban soils

\section{Conclusions}

(1) $\sum$ PAEs content reached $13.77(0.29-119.72) \mathrm{mg} / \mathrm{kg}, \mathrm{DBP}$ and DEHP were the primary PAE pollutants in the Changji urban soil. Generally, PAE concentrations were higher in urban soils than in agricultural soils. As PAEs in an urban environment are more complex and are emitted in greater volumes and and higher organic matter concentration, these primary might be the reason that the higher soil PAE content in urban soils.

(2) PAEs had obvious seasonal variations with the highest concentrations occurring in winter, followed by autumn and spring, while the lowest concentrations occurred in summer. The climatic characteristics of arid regions and the seasonal variation of emission sources were the primary influencing factors. Additionally, seasonal 
variations in PAEs in urban soils were not consistent with those in agricultural soils, which were primarily due to the different soil PAEs sources.

(3) The average non-carcinogenic risk values of the three PAEs were all less than one. However, the risk value of DEHP and DBP were relatively higher. The non-cancer risk of PAEs to children was higher than the risk to adults by approximately an order of magnitude. DEHP average carcinogenic risk values were lower than $10^{-6}$, but the average carcinogenic risk of DEHP to children was much higher than the risk to adults. All the theoretical values of the non-carcinogenic and carcinogenic risk of PAEs were within the acceptable safety range. Nevertheless, PAEs may still be harmful to the health of children and other susceptible populations.

\section{Acknowledgements}

This research was supported by National Natural Science Foundation (41867060), Key Research and Development Program of Hainan Province (ZDYF2019131). Thank for the Chemistry and Applied Chemistry of Changji University and School of Geography and Environmental science of Hainan Normal University for their guidance on the analysis as well as Shi Junlin, Ding Li, Zhang Bo, Zhu Shuyi and the other students who assisted with the sampling work.

\section{References}

Al-Natsheh M., Alawi M., Fayyad M. and Tarawneh I. (2015), Simultaneous GC-MS determination of eight phthalates in total and migrated portions of plasticized polymeric toys and childcare articles, Journal of Chromatography B, 985, 103109

Blount B.C., Milgram K.E., Silva M.J., Malek N.A., Reidy J.A., Needham L.L. and Brock J.W. (2000), Quantitative detection of eight phthalate metabolites in human urine using HPLCAPCI-MS/MS. Analytical Chemistry, 72(17), 4127-4134.

Bui T.T., Giovanoulis G., Cousins A.P., Magnér J., Cousins I.T. and de Wit C.A. (2016), Human exposure, hazard and risk of alternative plasticizers to phthalate esters, Science of the Total Environment, 541, 451-467.

Buzek J. and Ask B. (2009), Regulation (EC) No 1223/2009 of the European Parliament and of the Council of 30 November 2009 on cosmetic products, Official Journal of the European Union L 342 .

Chang C. (2011), The Globally Harmonized System of Classification and Labelling of Chemicals.

Duan Y., Meng X., Yang C., Pan Z., Chen L., Yu R. and Li F. (2010), Polybrominated diphenyl ethers in background surface soils from the Yangtze River Delta (YRD), China: occurrence, sources, and inventory, Environmental Science and Pollution Research 17(4), 948-956.

Hu X., Han Z.h., Liu B., Zhang F., Li F. and Wan W. (2007), Toxicity of Phthalate Esters (PAEs) and their distribution in the environment. Environmental Science and Management, 32(1), 37-40.

Kong L., Kadokami K., Wang S., Duong H.T. and Chau H.T.C. (2015), Monitoring of 1300 organic micro-pollutants in surface waters from Tianjin, North China, Chemosphere, 122, 125-130.

Kranich S.K., Frederiksen H., Andersson A.-M. and Jørgensen N. (2013), Estimated daily intake and hazard quotients and indices of phthtalate diesters for young danish men, Environmental Science \& Technology, 48(1), 706-712.

Langer S., Weschler C.J., Fischer A., Bekö G., Toftum J. and Clausen G. (2010), Phthalate and PAH concentrations in dust collected from Danish homes and daycare centers, Atmospheric Environment, 44(19), 2294-2301.

Li B., Wu S., Liang J., Liang W., Chen G., Li Y. and Yang G. (2015), Pollution Characteristics of Phthalate Esters (PAEs) in Soil and Agricultural Products in Agricultural Area of, Environmental Science, (6), 2283-2291.

Li H., Song W., Zhang Z., Ma W., Gao C., Li J., Huo C., Mohammed M.O., Liu L. and Kannan K. (2016), Phthalates in dormitory and house dust of northern Chinese cities: Occurrence, human exposure, and risk assessment, Science of the Total Environment, 565, 496-502.

Li Y. and Liu M. (2007), Study on Soil Properties and Pollution of Urumqi City, Arid Zone Research, 24(1), 66-69.

Maxwell C., Nelson D. and Masser C. (1978), Development of lead emission factor for reentrained dust from paved roadways, http://www.osti.gov/scitech/biblio/6032958.

Niu L., Yang X., Xu C., Yun L. and Liu W. (2014), Status of phthalate esters contamination in agricultural soils across China and associated health risks, Environmental Pollution, 195C, 16-23.

Paluselli A., Aminot Y., Galgani F., Net S. and Sempere R. (2018), Occurrence of phthalate acid esters (PAEs) in the northwestern Mediterranean Sea and the Rhone River, Progress in Oceanography, 3, 221-231.

Polezer G., Tadano Y.S., Siqueira H.V., Godoi A.F.L., Yamamoto C.I., de André P.A., Pauliquevis T., Andrade M.F., Oliveira A., Saldiva P.H.N., Taylor P.E. and Godoi R.H. (2018), Assessing the impact of PM2.5 on respiratory disease using artificial neural networks, Environmental Pollution, 235, 394-403.

Sakhi A.K., Lillegaard I.T.L., Voorspoels S., Carlsen M.H., Løken E.B., Brantsæter A.L., Haugen M., Meltzer H.M. and Thomsen C. (2014), Concentrations of phthalates and bisphenol A in Norwegian foods and beverages and estimated dietary exposure in adults, Environment International, 73, 259-269.

Sampson J. and De Korte D. (2011), DEHP-plasticised PVC: relevance to blood services, Transfusion Medicine, 21(2), 73-83.

Sun J., Pan L., Zhan Y., Lu H., Tsang D.C., Liu W., Wang X., Li X. and Zhu L. (2016), Contamination of phthalate esters, organochlorine pesticides and polybrominated diphenyl ethers in agricultural soils from the Yangtze River Delta of China, Science of the Total Environment, 544, 670-676.

USEPA (1996), Di(2-ethylhexyl) phthalate (DEHP), http://www.ep a.gov/iris/subst/0038.htm.

USEPA (2002), Dibutyl phthalate (DBP), http://www.epa.gov/ iris/subst/0038.htm.

USEPA (2004), Example Exposure Scenarios, National Center for Environmental Assessment U.S., U.S. Environmental Protection Agency Washington, DC 20460.

USEPA (2011), Exposure Factors Handbook 2011 Edition (Final). National Center for Environmental Assessment, Office of Research and Development, U.S. Environmental Protection Agency, Washington, DC, 20460.

USEPA (2013), Mid Atlantic Risk Assessment. Regional Screening Level (RSL) Summary Table. United States Environmental Protection Agency, Washington DC.

Wang J., Bo L., Li L., Wang D., Chen G., Christie P. and Teng Y. (2014), Occurrence of phthalate esters in river sediments in 
areas with different land use patterns, Science of the Total Environment, 500, 113-119.

Wang J., Chen G., Christie P., Zhang M., Luo Y. and Teng Y. (2015b), Occurrence and risk assessment of phthalate esters (PAEs) in vegetables and soils of suburban plastic film greenhouses, Science of the Total Environment, 523, 129-137.

Wang P. (2015), Spatiotemporal distribution of phthalic acid esters in protected agricultural soils in the black soil region of Northeast China, Northeast Agricultural University.

Wang Y., Shen C., Shen Z., Zhang D. and Crittenden J.C. (2015a), Spatial variation and sources of polycyclic aromatic hydrocarbons (PAHs) in surface sediments from the Yangtze Estuary, China, Environmental Science Processes \& Impacts, 17(7), 1340.

Xia X., Yang L., Bu Q. and Liu R. (2011), Levels, distribution, and health risk of phthalate esters in urban soils of Beijing, China, Journal of Environmental Quality, 40(5), 1643-1651.

Yang F. (2013), Experimental Study on Adsorption Behavior of Phthalate Compounds in Soil and QSPR Study, Nanjing University.

Zeng F., Cui K., Xie Z., Wu L., Luo D., Chen L., Lin Y., Liu M. and Sun G. (2009), Distribution of phthalate esters in urban soils of subtropical city, Guangzhou, China, Journal of Hazardous Materials, 164(2-3), 1171-1178.

Zhu Y., Tian J., Jing L., Wu G. and Wei F. (2012), Characteristics of Contamination of Phthalate Esters in Soil of Different Urban Functional Areas, Environmental Science \& Technology, 35(5), 42-46. 